\title{
Design and Methodology for the Korean Observational and Escitalopram Treatment Studies of Depression in Acute Coronary Syndrome: K-DEPACS and EsDEPACS
}

\author{
Jae-Min Kim ${ }^{\bowtie}$, Kyung-Yeol Bae', Hee-Ju Kang', Sung-Wan Kim¹, Il-Seon Shin', Young-Joon Hong², \\ Ju-Han Kim², Hee-Young Shin ${ }^{3}$, YoungKeun Ahn², Jong-Keun Kim4, Myung-Ho Jeong², and Jin-Sang Yoon ${ }^{1}$ \\ 'Department of Psychiatry, Chonnam National University Medical School, Gwangju, Republic of Korea \\ ${ }^{2}$ Department of Cardiology, Chonnam National University Medical School, Gwangju, Republic of Korea \\ ${ }^{3}$ Department of Biomedical Science, Chonnam National University Medical School, and Clinical Trial Center, Chonnam National University Hospital, \\ Gwangju, Republic of Korea \\ ${ }^{4}$ Department of Pharmacology, Chonnam National University Medical School, and Division of Clinical Pharmacology, Chonnam National University \\ Hospital, Gwangju, Republic of Korea
}

\begin{abstract}
Depression is common after acute coronary syndrome (ACS), adversely affecting cardiac course and prognosis. There have been only a few evidence-based treatment options for depression in ACS. Accordingly, we planned the Korean Depression in ACS (K-DEPACS) study, which investigated depressive disorders in patients with ACS using a naturalistic prospective design, and the Escitalopram for DEPACS (EsDEPACS) trial, which assessed the efficacy and safety of escitalopram for treating major or minor depression in patients with ACS. Participants in the K-DEPACS study were consecutively recruited from patients with ACS who were recently hospitalized at Chonnam National University Hospital, Gwangju, South Korea. Diagnoses were confirmed by coronary angiography from 2005. Data on depressive and cardiovascular characteristics were obtained at 2 weeks, 3 months, 12 months, and every 6 months thereafter following the index ACS admission. The K-DEPACS participants who met the DSM-IV criteria for major or minor depressive disorder were randomly assigned to groups in the 24-week, double-blind, placebo-controlled EsDEPACS trial beginning in 2007. The outcome of treatments for depressive and other psychiatric symptoms, issues related to safety, including general adversity, and cardiovascular factors were assessed. The K-DEPACS study can significantly contribute to research on the complex relationships between depression and ACS. The results of the EsDEPACS trial provide an additional treatment option for clinicians treating these patients.

Psychiatry Investig 2014;11:89-94
\end{abstract}

Key Words Acute coronary syndrome, Depression, Observational study, Clinical trial, Escitalopram.

\section{INTRODUCTION}

\section{Relationships between depression and acute coronary syndrome}

Depression is common in acute coronary syndrome [ACS; including myocardial infarction (MI) or unstable angina (UA)]. The prevalence of major depression was estimated to range from $15 \%$ to $27 \%{ }^{1}$ Furthermore, the occurrence of depressive symptoms after ACS has been associated with higher mor-

Received: January 28, 2013 Revised: April 1, 2013

Accepted: April 2, 2013 Available online: January 21, 2014

$\triangle$ Correspondence: Jae-Min Kim, MD, PhD

Department of Psychiatry, Chonnam National University Medical School, 160 Baekseo-ro, Dong-gu, Gwangju 501-746, Republic of Korea

Tel: +82-62-220-6143, Fax: +82-62-225-2351

E-mail:jmkim@chonnam.ac.kr

(a) This is an Open Access article distributed under the terms of the Creative Commons Attribution Non-Commercial License (http://creativecommons.org/licenses/by$\mathrm{nc} / 3.0$ ) which permits unrestricted non-commercial use, distribution, and reproduction in any medium, provided the original work is properly cited. bidity and mortality rates. Major depression was found to be the greatest predictor of the cardiac prognosis of patients with ACS, accounting for more than a twofold risk of developing an adverse cardiac complication. ${ }^{2}$

\section{Pharmacological trials for depression in ACS}

A number of randomized controlled trials, mostly with selective serotonin reuptake inhibitors (SSRIs), have examined the impact of pharmacological interventions on depression in patients with ACS. However, the trials conducted addressing this theory have produced mixed results. Fluoxetine was shown to be effective and safe for treating patients with post-MI depression. ${ }^{3}$ However, the limitations of this study were the small sample $(\mathrm{n}=54)$ and short treatment period (9 weeks) used to draw a conclusion. The Sertraline Antidepressant Heart Attack Randomized Trial (SADHART) involved 369 patients with major depression who were hospitalized with ACS 
and randomly assigned to receive sertraline or placebo for 24 weeks. ${ }^{4}$ Sertraline was found to be safe in these patients, but the overall efficacy results were less convincing. There was a restricted benefit of sertraline over placebo for the subgroup of patients with recurrent depression and those with a more severe depression. Mirtazapine was studied as a part of larger Myocardial Infarction and Depression-Intervention Trial (MIND-IT) that used a 24-week placebo-controlled design with 91 patients with major and minor depression post-MI. ${ }^{5}$ Although mirtazapine was shown to be safe and effective for achieving secondary outcomes, the results were complicated by a negative finding about the primary outcome and the small number of patients completing the study trial $(n=40)$. Citalopram was found to be safe and superior to placebo in reducing depressive symptoms in a 12-week Canadian Cardiac Randomized Evaluation of Antidepressant and Psychotherapy Efficacy (CREATE) trial. ${ }^{6}$ However, the outcome of this study was drawn from a different population of patients with moderateto-severe depression at late stage after hospitalization (ranging from 3 weeks to 31 years) for cardiac reasons.

\section{Limitations of the previous studies}

First, previous clinical trials have focused primarily on major depressive disorders. ${ }^{7}$ However, minor depressive disorders are even more common than major depressive disorders in patients with ACS, ${ }^{1,8}$ and these have negative effects on cardiac prognosis. ${ }^{9,10}$ Therefore, clinical trials are needed to evaluate the effect of minor depressive disorders in ACS. Second, previous randomized controlled trials have reported that serotonin selective reuptake inhibitors (SSRIs) were safe, but their antidepressant effects on patients with ACS were inconclusive because of small samples, low completion rates, controversial results, or heterogeneous study populations. Clinicians need more effective treatment options for these patients.

\section{The K-DEPACS and EsDEPACS studies}

We designed the Korean Depression in Acute Coronary Syndrome (K-DEPACS) to complement previous studies. The present study investigated depressive disorders in patients with ACS using a naturalistic prospective design. Patients with a diagnosis of a major or minor depressive disorder were enrolled in a 24-week, double-blind, placebo-controlled trial to assess the efficacy and safety of Escitalopram for Depression in ACS (EsDEPACS). The trial is registered at www.clinicaltrials.gov, registry number: NCT00419471. Escitalopram was chosen because of its antiplatelet effects in depressed patients, ${ }^{11}$ and it was safe and well tolerated in clinically non-depressed patients with a recent ACS diagnosis who were treated for 1 year. ${ }^{12}$ However, whether this drug is effective for treating depression with ACS remains unproven. Both K-DEPACS and
EsDEPACS are investigator-initiated and designed studies.

The K-DEPACS study investigated the occurrence, risk factors, and longitudinal course of psychological disorders (depression in particular) in survivors of recently developed ACS as well as the effects of depression on cardiac course and prognosis. The EsDEPACS study evaluated the efficacy and safety of escitalopram in the treatment of depressed patients with ACS and determined the effects of escitalopram on other psychiatric outcomes, including social functioning, disability, and quality of life.

\section{METHODS}

\section{Study outline and recruitment}

The outlines of the K-DEPACS and EsDEPACS studies are presented in Figure 1. The K-DEPACS study investigated the epidemiology of depressive disorders in patients with ACS using a naturalistic prospective design and has been supported by the Korean Ministry of Health and Welfare since 2005. Participants were consecutively recruited from patients recently hospitalized with ACS; diagnosis was confirmed by coronary angiography and laboratory examinations performed at the Department of Cardiology of Chonnam National University Hospital, Gwangju, Korea. Among patients who met eligibility criteria and agreed to participate, psychiatric assessments were made at 2 weeks, 3 months, 12 months, and every 6 months thereafter following ACS diagnosis to investigate consequences of ACS at acute, subacute, and chronic stages. Potential participants in the EsDEPACS study were screened for depressive symptoms with the Beck Depression Inventory $(\mathrm{BDI})^{13}$ during hospitalization at 2 weeks after ACS diagnosis and, at an outpatient clinic, every 1 month thereafter up to 3 months. Patients with depressive symptoms (BDI $>10)$ were clinically evaluated by the study psychiatrists using the Mini-International Neuropsychiatric Interview (MINI), a structured diagnostic psychiatric interview using DSM-IV criteria to diagnose major or minor depressive disorder. ${ }^{14}$ Beginning in 2007, patients with a diagnosis of major or minor depressive disorder who agreed to participate were randomly assigned to the EsDEPACS study, which was, supported by H. Lundbeck A/S (Copenhagen, Denmark). Patients who were not depressed or who were depressed but did not want to participate in the EsDEPACS study received conventional treatment for ACS and were evaluated with the K-DEPACS protocol.

\section{Study subjects}

All patients who visited the study site with angina symptoms and were hospitalized were approached for enrollment in the K-DEPACS study at 2 week after the index ACS. Patients who met the depression criteria in that hospitalized setting or at an 
Figure 1. Flow chart of the K-DEPACS and EsDEPACS studies. BDI: Beck Depression Inventory, ACS: acute coronary syndrome, K-DEPACS: Korean Depression in Acute Coronary Syndrome study, MINI: Mini-International Neuropsychiatric Interview, EsDEPACS: Escitalopram for Depression in Acute Coronary Syndrome.

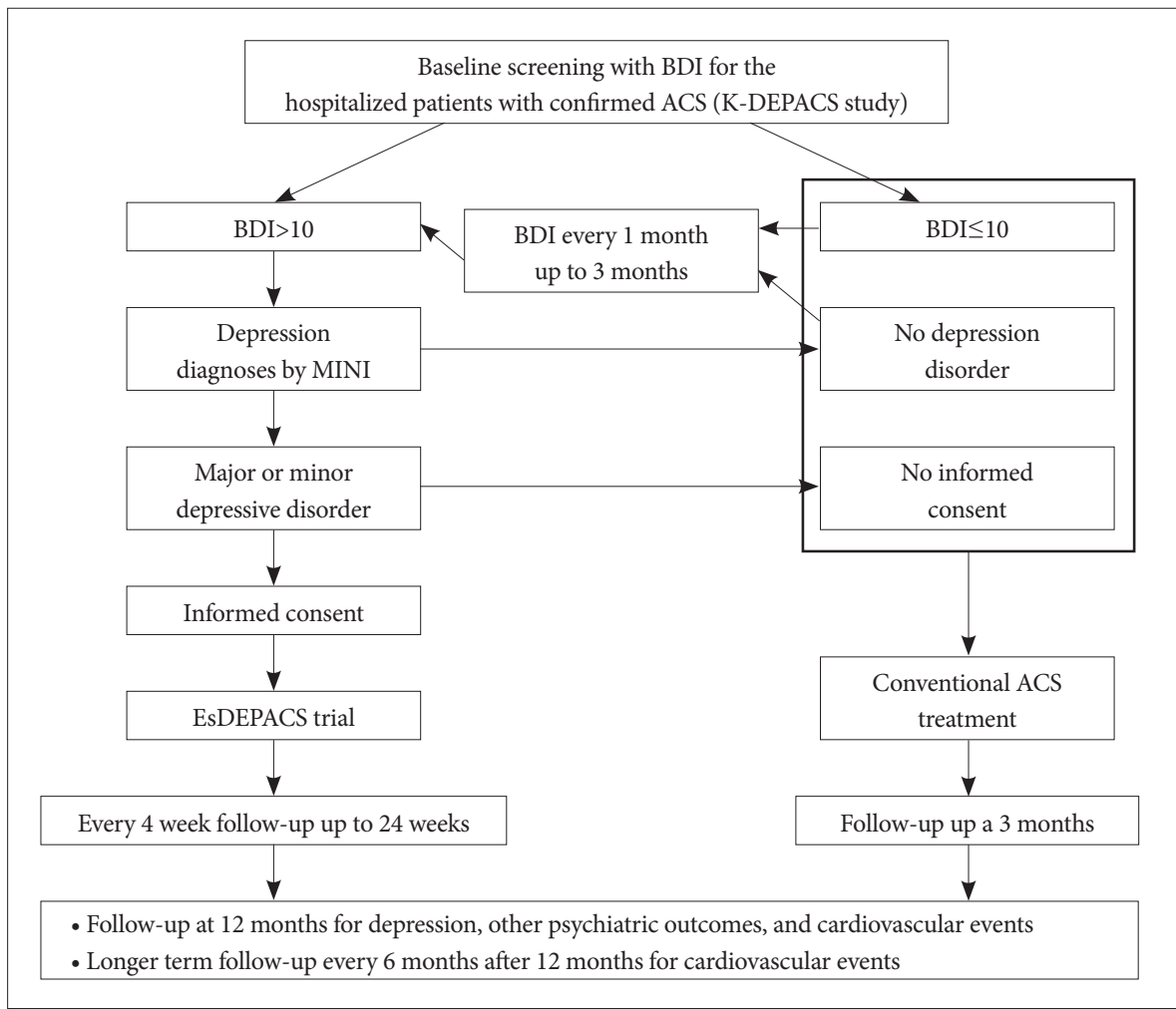

outpatient setting, every 1 month thereafter up to 3 months were qualified for inclusion in the EsDEPACS study. Inclusion and exclusion criteria for both studies are summarized in Table 1.

\section{Assessment and measurements for the K-DEPACS study}

General information on socio-demographic characteristics and health status were obtained. Cardiovascular medications were recorded. Measurements of depressive symptoms included the 17-item Hamilton Depression Rating Scale (HAMD), ${ }^{15}$ the Montgomery-Asberg Depression Rating Scale (MADRS), ${ }^{16}$ the BDI, and the Clinical Global Impression Scaleseverity (CGI-s). ${ }^{17}$ We used four scales because they differed from one another in several ways: the HAMD is the observerrated scale most widely used in research on depression related to $\mathrm{ACS}^{18}$; the MADRS (another observer-rated scale) does not include items for evaluating the somatic symptoms of depression, which may be difficult to differentiate from the physical consequences of ACS; the BDI is a self-report measure; and the CGI-s assesses global symptomatology and can be used quickly in a busy clinical setting. Scales used to assess other psychiatric symptoms included the Social and Occupational Functioning Assessment Scale (SOFAS), ${ }^{19}$ the World Health Organization Disability Assessment Schedule-12 (WHODAS-12), ${ }^{20}$ and the World Health Organization Quality of Life Scale-abbreviated form (WHOQOL-BREF). ${ }^{21}$ We included these measures given recent recommendations that depres- sion outcome research involve multifaceted evaluation (i.e., more than only the HAMD) to address psychological wellbeing and functioning. ${ }^{22}$ Additionally, personality was assessed at the 3-month evaluation point using the Big Five Inventory (BFI). ${ }^{23}$ All assessment scales had been formally translated and standardized in Korean. ${ }^{24-30}$ Cardiac risk factors included self-reported diagnoses of and treatment histories for hypertension and diabetes mellitus; resting blood pressure (BP); fasting cholesterol levels; body mass index; and current smoking status. Coronary angiography results were used for diagnoses of MI or UA. Echocardiography results included left ventricular ejection fraction (LVEF) and wall motion scores. Electrocardiography (ECG) variables were heart rate (beats/ min), PR interval (ms), QRS duration (ms), and QTc duration (ms). Laboratory tests for serum cardiac biomarkers troponin I and creatine kinase-MB (CK-MB) were measured. New cardiovascular events, including angina, heart failure, or death, were recorded.

\section{The EsDEPACS trial}

\section{Randomization and intervention}

The efficacy and safety of flexible doses of escitalopram (5 $\mathrm{mg}, 10 \mathrm{mg}, 15 \mathrm{mg}$, or $20 \mathrm{mg}$ ) were investigated using a double-blind, placebo-controlled design. The escitalopram and placebo were provided by $\mathrm{H}$. Lundbeck A/S (Copenhagen, Denmark). Patients were randomized in a $1: 1$ ratio for as- 
Table 1. Selection criteria

\begin{tabular}{|c|c|c|}
\hline & K-DEPACS study & EsDEPACS study \\
\hline $\begin{array}{l}\text { Inclusion } \\
\text { criteria }\end{array}$ & $\begin{array}{l}\text { Aged 18-85 } \\
\text { Confirmed diagnoses as ACS (acute MI or UA) by coronary } \\
\text { angiography } \\
\text { With ability to complete various questionnaires } \\
\text { Can understand the objective of the study and sign informed } \\
\text { consent }\end{array}$ & $\begin{array}{l}\text { Same as K-DEPACS study plus } \\
\text { BDI }>10 \\
\text { DSM-IV major or minor depressive disorder by the MINI }\end{array}$ \\
\hline $\begin{array}{c}\text { Exclusion } \\
\text { criteria }\end{array}$ & $\begin{array}{l}\text { Occurrence of ACS while the patient was hospitalized for } \\
\text { another reason, except for ACS } \\
\text { Current ACS developed less than } 3 \text { months after coronary } \\
\text { artery bypass graft procedure } \\
\text { Uncontrolled hypertension (systolic BP) } \\
>180 \mathrm{~mm} \mathrm{Hg} \text { or diastolic BP }>100 \mathrm{~mm} \mathrm{Hg} \\
\text { Resting heart rate }<40 / \mathrm{min} \\
\text { Severe physical illnesses threatening life or interfering with } \\
\text { the recovery from ACS } \\
\text { Persistent clinically significant laboratory abnormalities }\end{array}$ & $\begin{array}{l}\text { Same as K-DEPACS study plus } \\
\text { Concomitant use of class I antiarrhythmics; reserpine, } \\
\text { guanethidine, clonidine, or methyldopa; lithium, } \\
\text { anticonvulsants or neuroleptics; antidepressants } \\
\text { History of neuropsychiatric illnesses such as dementia, } \\
\text { Parkinson's disease, brain tumor, psychoses, bipolar disorder, } \\
\text { alcoholism, and other substance dependence } \\
\text { Pregnancy } \\
\text { Participating other drug trials }\end{array}$ \\
\hline
\end{tabular}

K-DEPACS: Korean Depression in Acute Coronary Syndrome study, EsDEPACS: Escitalopram for Depression in Acute Coronary Syndrome, ACS: acute coronary syndrome, MI: myocardial infarction, UA: unstable angina, BDI: Beck Depression Inventory, DSM-IV: Diagnostic and Statistical Manual, 4th edition, MINI: Mini-International Neuropsychiatric Interview, BP: blood pressure

signment into the ecsitalopram or placebo group according to computer-generated randomization codes. Patient visits were scheduled at baseline and at 4, 8, 12, 16, 20, and 24 weeks ( \pm 7 -day visit window was allowed) thereafter. Patients received one tablet of escitalopram $5 \mathrm{mg}, 10 \mathrm{mg}$, or $20 \mathrm{mg}$ or two tablets of escitalopram ( $5 \mathrm{mg}$ plus $10 \mathrm{mg}$ ) for a 15-mg dose per day or a matched placebo. The initial dose at baseline evaluation was typically $10 \mathrm{mg} /$ day, but subjects aged 65 years or over and those with hepatic dysfunction received $5 \mathrm{mg} /$ day. After the second evaluation (week 4), the medication doses could be changed and were determined by the investigators' clinical judgment based on the response to and tolerability of treatment. Medications were taken orally once daily within 30 min after supper. Adherence to the medication regimen was confirmed by pill counts at every visit and was defined as acceptable when at least $75 \%$ of the medication was taken. At the end of 24 weeks of double-blind treatment, the study was completed, and study medication was tapered. To ensure patient safety, follow-up treatment was provided at the outpatient clinic of the psychiatric department when the clinician determined a need for further treatment. Concomitant medications, such as any other antidepressants, psychostimulants, antipsychotics, and anticholinergics, were not permitted. However, transient use of analgesics, antipyretics, and cold medicines, as well as hypnotics such as zolpidem, triazolam, and benzodiazepines, was allowed.

\section{Outcome measures}

The primary efficacy outcome measure was the HAMD.
The secondary depression outcome measures were the MADRS, BDI, and CGI-s. Other psychiatric outcome measures included the SOFAS, WHODAS-12, WHOQOL-BREF, and Chonnam National University Hospital-Leeds Sleep Evaluation Questionnaire (CNUH-LSEQ). ${ }^{31}$ The HAMD, MADRS, BDI, CGI-s, and CNUH-LSEQ were administered at baseline and every follow-up visit; the SOFAS, WHODAS-12, and WHOQOL-BREF were assessed at baseline and at follow-up weeks 4,12 , and 24 . The BFI was administered at follow-up week 12. In terms of general safety outcomes, adverse events during the study period were recorded at all visits. Serious clinical and laboratory adverse events were assessed. Discontinuation of participation in the study due to adverse events was recorded. In terms of cardiovascular safety outcomes, results on echocardiography (LVEF and wall motion), ECG (heart rate, PR interval, QRS duration, and QTc duration), laboratory tests (troponin I, CK-MB, and total cholesterol), and body weight were measured at baseline and at last followup visit. Resting BP was measured at baseline and every follow-up visit. After finishing the EsDEPACS trial, all participants, regardless of completion status, were approached regarding participation in the K-DEPACS long-term follow-up evaluations scheduled at 12 months and every 6 months thereafter (Figure 1).

\section{Sample size}

The overall sample size was estimated from the number of subjects needed for the EsDEPACS trial. The ESDEPACS trial was designed to produce $80 \%$ power to detect small to medi- 
um effect sizes (0.35) on the primary efficacy outcome for both treatments (i.e., a mean score difference of 2 on the HAMD assuming a SD of 5-6) in the ITT analyses for all randomized participants when performing two-sided tests at $\alpha=$ 0.05 . These assumptions yielded a sample size of 106 patients per group. As a previous study reported that a considerable proportion of Korean patients who were depressed (30\%) discontinued the study after the baseline evaluation, ${ }^{32}$ we decided to enroll a total of 300 patients with ACS who were also depressed (150 subjects per group) for the EsDEPACS trial. We predicted that two-thirds of these patients would participate in the EsDEPACS study and that one-third may not want to participate. Therefore, approximately 450 depressed patients were needed for the EsDEPACS study. The prevalence of major depression reportedly ranges from $15 \%$ to $27 \%$ (approximately $20 \%$ ), and at least a comparable proportion of patients with ACS have minor depression. ${ }^{1}$ Based on these estimates, we predicted that $40 \%$ of the sample would meet criteria for major or minor depressive disorders. Accordingly, approximately 1125 patients with ACS were needed to provide a pool of 450 depressed patients and to enroll 300 in the EsDEPACS trial.

\section{CONCLUSION}

The K-DEPACS study is a meaningful and significant contribution to the research on the complex relationships between depression and ACS. The particular prospective naturalistic study design might facilitate other related studies and enhance the understanding on this issue. ${ }^{33}$ The EsDEPACS trial is the first to demonstrate the efficacy and safety of escitalopram for treating depression in ACS patients. Despite the high prevalence and negative effects of depression in ACS patients, only a few evidence-based options are currently available for the treatment of depression in these patients. We believe that the results of the EsDEPACS trial provide an additional treatment choice for clinicians who treat these patients.

\section{Acknowledgments}

This research was supported by a grant of the Korea Health $21 \mathrm{R} \& \mathrm{D}$, Ministry of Health and Welfare, Republic of Korea (HI10C2020) and an unrestricted research grant from $\mathrm{H}$. Lundbeck A/S. The funders had no role in study design; in the collection, analysis and interpretation of data; in the writing of the report; or in the decision to submit the paper for publication.

\section{REFERENCES}

1. Rudisch B, Nemeroff CB. Epidemiology of comorbid coronary artery disease and depression. Biol Psychiatry 2003;54:227-240.

2. Carney RM, Rich MW, Freedland KE, Saini J, teVelde A, Simeone C, et al. Major depressive disorder predicts cardiac events in patients with coronary artery disease. Psychosom Med 1988;50:627-633.

3. Strik JJ, Honig A, Lousberg R, Lousberg AH, Cheriex EC, Tuynman-
Qua HG, et al. Efficacy and safety of fluoxetine in the treatment of patients with major depression after first myocardial infarction: findings from a double-blind, placebo-controlled trial. Psychosom Med 2000; 62:783-789.

4. Glassman AH, O’Connor CM, Califf RM, Swedberg K, Schwartz P, Bigger JT Jr, et al. Sertraline treatment of major depression in patients with acute MI or unstable angina. JAMA 2002;288:701-709.

5. Honig A, Kuyper AM, Schene AH, van Melle JP, de Jonge P, Tulner $\mathrm{DM}$, et al. Treatment of post-myocardial infarction depressive disorder: a randomized, placebo-controlled trial with mirtazapine. Psychosom Med 2007;69:606-613.

6. Lespérance F, Frasure-Smith N, Koszycki D, Laliberté MA, van Zyl LT, Baker B, et al. Effects of citalopram and interpersonal psychotherapy on depression in patients with coronary artery disease: the Canadian Cardiac Randomized Evaluation of Antidepressant and Psychotherapy Efficacy (CREATE) trial. JAMA 2007;297:367-379.

7. Dowlati Y, Herrmann N, Swardfager WL, Reim EK, Lanctot KL. Efficacy and tolerability of antidepressants for treatment of depression in coronary artery disease: a meta-analysis. Can J Psychiatry 2010;55:91-99.

8. Jiang W, Krishnan RR, O'Connor CM. Depression and heart disease: evidence of a link, and its therapeutic implications. CNS Drugs 2002; 16:111-127.

9. Bush DE, Ziegelstein RC, Tayback M, Richter D, Stevens S, Zahalsky $\mathrm{H}$, et al. Even minimal symptoms of depression increase mortality risk after acute myocardial infarction. Am J Cardiol 2001;88:337-341.

10. Rallidis LS, Varounis C, Sourides V, Charalampopoulos A, Kotakos C, Liakos G, et al. Mild depression versus C-reactive protein as a predictor of cardiovascular death: a three year follow-up of patients with stable coronary artery disease. Curr Med Res Opin 2011;27:1407-1413.

11. Flöck A, Zobel A, Bauriedel G, Tuleta I, Hammerstingl C, Höfels S, et al. Antiplatelet effects of antidepressant treatment: a randomized comparison between escitalopram and nortriptyline. Thromb Res 2010; 126:e83-e87.

12. Hanash JA, Hansen BH, Hansen JF, Nielsen OW, Rasmussen A, BirketSmith M. Cardiovascular safety of one-year escitalopram therapy in clinically nondepressed patients with acute coronary syndrome: results from the DEpression in patients with Coronary ARtery Disease (DECARD) trial. J Cardiovasc Pharmacol 2012;60:397-405.

13. Beck AT, Ware CH, Mendelson M, Mock J, Erbaugh J. An inventory for measuring depression. Arch Gen Psychiatry 1961;4:561-571.

14. Sheehan DV, Lecrubier Y, Sheehan KH, Amorim P, Janavs J, Weiller E, et al. The mini-international neuropsychiatric interview (M.I.N.I): the development and validation of a structured diagnostic psychiatric interview for DSM-IV and ICD-10. J Clin Psychiatry 1998;59(Suppl 20): 22-33.

15. Hamilton M. A rating scale for depression. J Neurol Neurosurg Psychiatry 1960;23:56-62.

16. Montgomery SA, Asberg M. A new depression scale designed to be sensitive to change. Br J Psychiatry 1979;134:382-389.

17. Guy W. ECDEU Assessment Manual for Psychopharmacology. Revised. Rockville: National Institute of Mental Health; 1976.

18. Davidson KW, Kupfer DJ, Bigger JT, Califf RM, Carney RM, Coyne JC, et al. Assessment and treatment of depression in patients with cardiovascular disease: National Heart, Lung, and Blood Institute Working Group Report. Psychosom Med 2006;68:645-650.

19. American Psychiatric Association. Diagnostic and Statistical Manual of Mental Disorders, 4th Edition. Washington, DC: American Psychiatric Association; 1994.

20. Eipping-Jordan J, Ustun TB, Assessment, Classification and epidemiology Group. The WHODAS II: levelling the playing field for all disorders. WHO Mental Health Bull 2000;6:5-6.

21. Development of the World Health Organization WHOQOL-BREF quality of life assessment. The WHOQOL Group. Psychol Med 1998; 28:551-558.

22. Fava GA, Ruini C, Belaise C. The concept of recovery in major depres- 
sion. Psychol Med 2007;37:307-317.

23. John OP, Srivastata S. The Big Five Trait taxonomy: History, Measurement, and Theoretical Perspectives. In: Pervin LA, John OP, Editors. Handbook of Personality, 2nd Edition. New York: The Guilford Press; 1999, p.102-138.

24. Ahn YM, Lee KY, Yi JS, Kang MH, Kim DH, Kim JL, et al. A validation study of the Korean-version of the Montgomery-Asberg Depression Rating Scale. J Korean Neuropsychiatr Assoc 2005;44:466-476.

25. Chung YC, Rhee MK, Lee YH, Park SH, Shon CH, Lee BK, et al. Standardization study of Beck Depression Inventory 1-Korean version (KBDI): reliability and factor analysis. Korean J Psychopathol 1995;4:77-95.

26. Kim JM, Stewart R, Glozier N, Prince M, Kim SW, Yang SJ, et al. Physical health, depression and cognitive function as correlates of disability in an older Korean population. Int J Geriatr Psychiatry 2005;20:160-167.

27. Lee JY, Cho MJ, Kwon JS. Global Assessment of Functioning Scale and Social and Occupational Functioning Scale. Korean J Psychopharmacol 2006;17:122-127.

28. Yi JS, Bae SO, Ahn YM, Park DB, Noh KS, Shin HK, et al. Validity and reliability of the Korean version of the Hamilton Depression Rating
Scale (K-HDRS). J Korean Neuropsychiatr Assoc 2005;44:456-465.

29. Yoo SW, Kim YS, Noh JS, Oh KS, Kim CH, Namkoong K, et al. Validity of Korean version of the Mini-International Neuropsychiatric Interview. Anxiety Mood 2006;2:50-55.

30. Kim SY, Kim JM, Yoo JA, Bae KY, Kim SW, Yang SJ, et al. Standarization and validation of Big Five Inventory-Korean Version (BFI-K) in elders. Korean J Biol Psychiatry 2010;17:15-25.

31. Yoon JS, Yoon BH, Choi TS, Kim YB, Lee HY. A double-blind comparison of paroxetine and amitriptyline in the treatment of depression accompanied by alcoholism: behavioral side effects during the first 2 weeks of treatment. J Korean Soc Biol Psychiatry 1996;3:277-287.

32. Kim JM, Kim SW, Stewart R, Kim SY, Yoon JS, Jung SW, et al. Predictors of 12-week remission in a nationwide cohort of people with depressive disorders: the CRESCEND study. Hum Psychopharmacol 2011;26:41-50.

33. Marks DM, Thanasellan J, Pae CU. Innovations in clinical research design and conduct in psychiatry: shifting to pragmatic approaches. Psychiatry Investig 2009;6:1-6. 